\title{
Wohnungsleerstand, Hochschuleffekte und ländliche Infrastrukturversorgung - Spannungsfelder räumlicher Entwicklung
}

\author{
Gregor Prinzensing
}

Online publiziert: 28. September 2015

(C) Springer-Verlag Berlin Heidelberg 2015

Liebe Leserinnen und Leser,

auch die vorliegende Ausgabe von „Raumforschung und Raumordnung" enthält wieder einige neue und lesenswerte Ergebnisse aktueller raumwissenschaftlicher Forschung. Aus den Zufälligkeiten des Workflows bei der arbeitsteiligen Fertigstellung von Manuskripten heraus hat sich diese Ausgabe hinter dem Rücken aller Beteiligten zu einer Art Themenheft entwickelt. In drei von vier Artikeln dreht es sich (wenigstens auch) um ländlich gelegene bzw. dünn besiedelte Räume und deren Unterschiede zu anderen Teilräumen Deutschlands. Das Wirken der Hochschulen tritt als eine Art Nebenschwerpunkt hinzu - immerhin auch zweifacher Gegenstand der raumbezogenen Untersuchungen, über die wir Ihnen hiermit berichten.

Im ersten wissenschaftlichen Beitrag des Heftes untersuchen Johannes Glückler, Robert Panitz und Christian Wuttke im Rahmen einer regional und sektoral differenzierten Analyse „Die wirtschaftliche Wirkung der Universitäten im Land Baden-Württemberg“. Dazu wird die ökonomische Bedeutung der staatlichen Universitäten eines großen Flächenlandes in Deutschland umfassend ermittelt, indem eine Berechnung direkter, indirekter und induzierter Nachfrageund Ausgabeneffekte erfolgt. Dadurch wird der von den neun Landesuniversitäten ausgehende makroökonomische Einfluss in Bezug auf die Wertschöpfung und den Arbeitsmarkt des Bundeslandes abgeschätzt und monetär beziffert. Neben einer solchen Prüfung der regionalwirtschaftlichen Wertschöpfungs- und Beschäftigungseffekte stellen die Autoren auch einen Vergleich mit alternativen Veraus-

G. Prinzensing $(\square)$

Akademie für Raumforschung und Landesplanung (ARL), Leibniz-Forum für Raumwissenschaften,

Hohenzollernstraße 11,

30161 Hannover, Deutschland

E-Mail: prinzensing@arl-net.de gabungsmöglichkeiten des staatlichen Budgets an (eine sogenannte differenzielle Inzidenzanalyse). Nach Ansicht der Autoren zeigen die Analysen, dass die Universitäten Baden-Württembergs in ihrer Gesamtwirkung einen Beitrag zur regionalen Wertschöpfung und Beschäftigung leisten, der die zur Grundfinanzierung aufgewendeten Mittel des Landes nahezu verdoppelt; alternative Verwendungen dieser Mittel vermögen kaum eine vergleichbare Wirkung zu erzielen.

Dieter Rink und Manuel Wolff erforschen sodann in ihrem wissenschaftlichen Beitrag den „Wohnungsleerstand in Deutschland“ und zeigen Divergenzen der deutschen Wohnungsmarktentwicklung auf. Mithilfe von Ergebnissen der Gebäude- und Wohnungszählung (GWZ) 2011 werden die räumliche Disparität bei der Entwicklung des Wohnungsleerstandes dargestellt und ein breites Spektrum an Ursachen für seine Erklärung und Bewertung diskutiert. Darüber hinaus wird die große Varianz der kommunalen Leerstandsquoten gezeigt: Leerstand und Wohnungsknappheit existieren zum Teil räumlich und zeitlich dicht nebeneinander. Der Beitrag macht außerdem heuristische Vorschläge für eine systematische Kategorisierung von Leerstand und klassifiziert ihn sowohl unter dem Gesichtspunkt der Versorgung der Bevölkerung mit Wohnraum wie auch hinsichtlich der Marktgängigkeit beim Verkauf bzw. der Vermietung von Wohnungen. Das macht die dargestellten Überlegungen für die Städtebaupolitik wie die Immobilienwirtschaft bedeutsam. Die Autoren unternehmen damit den Versuch, für die verschiedenen Ausprägungen und Bewertungen der Leerstandsquote ein systematisches Konzept zu entwickeln. So könnte ein ihrer Ansicht nach unterbestimmter und bislang auch unterschätzter Indikator als langfristig nutzbare Größe eingeführt und künftig bei der Wohnungsmarktbeobachtung verwendet werden. 
In ihrem anschließenden Bericht aus Forschung und Praxis untersuchen Grit Rost, Kirsten Maier, Martin Böhm und Jörg Londong die „Auswirkungen eines technischen Paradigmenwechsels auf die wasserwirtschaftliche Organisation in strukturschwachen ländlichen Räumen“. Sogenannte „Neuartige Sanitärsysteme“ (NASS) eröffnen ihrer Ansicht nach alternative Entwicklungsansätze für ländliche Räume. Vor allem in schrumpfenden Regionen führen demographischer Wandel und Klimawandel schon seit einigen Jahren dazu, dass bestehende Entsorgungssysteme - und zumal das konventionelle System der Zentralableitung von Abwässern - an technische und finanzielle Grenzen stoßen. Der Beitrag stellt vor diesem Hintergrund einen innovativen Versuch vor, wie für die zukünftige Gestaltung der Abwasserentsorgung in ländlichen Räumen flexible und an die jeweiligen lokalen Bedingungen besser angepasste Lösungen entwickelt werden können, die dazu noch einen Beitrag zur regionalen Wertschöpfung leisten. Nach Ansicht der Autoren kommt dieser Einsatz alternativer Technologien bei der Siedlungsentwässerung einem Paradigmenwechsel gleich, aus dem sich auch über den engeren Bereich der Abwasserentsorgung hinausreichende Chancen für die regionalwirtschaftliche Entwicklung strukturschwacher ländlicher Räume ergeben.

Im vierten Artikel schließlich betrachten Michael Fritsch und Matthias Piontek „Die Hochschullandschaft im demo- graphischen Wandel“ und verzeichnen „Entwicklungstrends und Handlungsalternativen“ (so der Untertitel) für die Universitäten und Fachhochschulen in verschiedenen Raumordnungsregionen Deutschlands. Hierfür werden Daten zu den Hochschulen vom Statistischen Bundesamt und Schätzungen zur Entwicklung der Studierendenzahlen unter anderem von der Kultusministerkonferenz (KMK) den regionalisierten demographischen Vorausberechnungen des Bundesinstituts für Bau-, Stadt- und Raumforschung (BBSR) für städtische Regionen, Regionen mit Verdichtungsansätzen und ländliche Räume bis 2030 gegenübergestellt. Den Ergebnissen zufolge werden die Hochschulen und Universitäten in der Zukunft offenbar sehr unterschiedlich vom demographischen Wandel betroffen sein. Das hängt nicht nur von ihrem Standort ab (städtisch oder ländlich gelegen), sondern auch von ihrer Größe und ihrem Status (Volluniversität oder Fachhochschule). Der Bericht verfolgt die These, dass aufgrund der zunehmenden räumlichen Mobilität der Studierenden kein nennenswerter Zusammenhang zwischen der Bevölkerungsentwicklung und der Anzahl der Studierenden in der jeweiligen Region herstellbar ist. Dem Wettbewerb zwischen den Hochschulen wird deshalb nach Ansicht der Autoren künftig noch eine wesentlich höhere Bedeutung zukommen.

Wir wünschen Ihnen bei den Texten wie immer eine kurzweilige und erkenntnisreiche Lektüre. 